\title{
Computing an organism: on the interface between informatic and dynamic processes
}

\author{
Paulien Hogeweg * \\ Theoretical Biology and Bioinformatics Group, Utrecht University, Padualaan 8, 3584, CH Utrecht, The Netherlands
}

Received 4 June 2001; received in revised form 14 July 2001; accepted 1 August 2001

\begin{abstract}
The importance of interactions between processes at diverse space and time scales in biological information processing is a recurrent theme in the work of Michael Conrad (BioSystems, 1995: 35, 157-160; BioSystems, 1999: 52, 99-100). In this paper I present some results of explicit computational models that aim to capture and exploit some of the essential features of such multi-level processes. In the model formulation, I try to minimize the explicit definition of inter-level interactions, while providing the possibility of such interactions to develop. As often argued by Conrad inter-level interactions limit programmability. Indeed, we use an evolutionary process to derive the specific models. We study morphogenesis. We show that the interplay between cell adhesion and cell differentiation provides interesting mechanisms for morphogenesis. We also show that the interplay can both reduce and enhance small random fluctuations. We show that unequal cell cleavage in the early embryo-genesis reduces inter-individual variation of the morphemes developed from the same 'genome'. Our results suggest that, during evolution, the interplay between levels is exploited while it is at the same time reduced so as to give a certain primacy to inherited information. (C) 2002 Elsevier Science Ireland Ltd. All rights reserved.
\end{abstract}

Keywords: Morphogenesis; Cell adhesion; Cell differentiation; Gene regulation; Evolution; Inter-level interaction

\section{Introduction}

'How to compute an organism': it is certainly open to dispute in what sense this sentence makes any sense. Indeed, an important theme in the work of Michael Conrad (1995, 1996), in whose honor and memory I write this paper, is the argument that because of percolation of micro-

* Tel.: + 31-30-2533692; fax: + 31-30-2513655.

E-mail address: p.hogeweg@bio.uu.nl (P. Hogeweg). scale physical processes, 'computing' an organism is impossible. Why then this provocative title? It is because indeed the interaction of processes at different space and time scales is the main theme of the work that I will discuss. Such interactions are a hallmark of biotic systems. Therefore, they should be explored and exploited in our attempts to model and understand processes in biotic systems.

A second reason for the title of this paper is Lee Segel's (Segel, 2001) commentary 'Computing an organism' about our model on Dictyostelium development, which is based on the interplay be- 
tween two well known and well characterized 'physical' processes, i.e. excitable media as model for long-range cAMP signaling, and cellular adhesion. In Section 4, I will shortly discuss how it is indeed the interplay between these processes that (literally) 'shapes' the organism in that model. Nevertheless, 'informatic' processes, in this case gene regulation, are (almost) fully absent in the model.

In Section 5, I will describe a model in which I explore the interplay between gene regulation and cell adhesion and the resulting morphogenetic processes. Another important theme in the work of Michael Conrad (e.g. Conrad, 1996) is the relation between evolvability and programmability. Indeed I will use, and we indeed do need, an evolutionary process in order to focus on generic features of (evolved) mechanism of morphogenesis that rely on the interplay between 'informatic' and 'physical' processes.

Finally, I will study how evolutionary processes on the one hand exploit and on the other hand may tend to reduce the interplay between 'uncontrolled' physical and evolved informatic processes.

\section{Classical models of development: pattern formation and morphogenesis}

In this section, I will shortly discuss two different classical approaches for studying morphogenesis in order to set the stage for models attempting to incorporate the interaction between different levels of description.

\subsection{Pattern formation in reaction diffusion systems}

Allegedly Turing said, when praised for his well known reaction diffusion mechanism for pattern formation (the so called Turing patterns), 'well, the stripes are easy, but what about the horse part?' Nevertheless, morphogenesis is still mostly modeled in terms of 'Turing patterns' and related reaction diffusion systems.

The appeal of such models is in their apparent simplicity (and therewith tractability) and their power to generate complex patterns, (which do sometimes resemble observed patterns) from almost nothing. Interestingly, the well studied system of stripe formation in the early embryogenesis of Drosophila is a striking counter example of this kind of simplicity in biological systems: each of the stripes is generated by a different regulatory mechanism. In hindsight: why not use the differences which are there anyway, especially since the stripes while being initially similar, should later on differentiate? For a recent paper exploring the (evolution of) hierarchical control versus uniform control see Salazar-Ciudad et al. (2001).

Moreover, a point to keep in mind is that although, 'diffusion' appears to be a simple process, in a cellular system, in which the diffusing substance has to enter the cell via receptors, it is not, as Kerszberg and Wolpert (1998) have shown. Nevertheless, both at intra- and inter-cellular scales, pattern formation by 'short range activation and long range inhibition' appears to be a powerful mechanism to enhance or to (re) generate spatial differentiation (for a recent review see Meinhardt and Gierer, 2000). Similarly, excitation waves (of e.g. Calcium, cAMP), can be modeled profitably by reaction diffusion systems. In my opinion, however, models of development and morphogenesis should preferably go a step beyond such a 'uniform' explanation, in which only the formation of a prepattern is addressed independent of the (always inhomogeneous) medium in which it takes place, and independent of the actual 'morphogenesis' that it is supposed to trigger. One reason is that although, the 'generic' patterns formed by these processes certainly do occur in biotic systems, an elephant is not such a generic pattern.

\subsection{Graftal patterns in ancestor based systems}

Lindenmayer (1968a,b, 1975) introduced parallel grammar-like rewriting systems (known as Lsystems) to describe (plant) development. In contrast to reaction diffusion systems, this formalism focuses on discrete cells that are in discrete states representing differences in gene expression. The next-state of a cell depends on its current state and the state of the neighbors. The next state rules are applied to all cells simultaneously. In 
addition to state changes cell divisions occur, giving rise to a new cell, which is subsequently subjected to the same rewriting rule. Thus, growth is an inherent feature of the system. The neighborhood relation in these systems is entirely 'ancestry based': the notion of space is not included in the original formulation. This feature provides the tractability of these actually 'developing' systems.

The structures to which these systems can give rise were termed 'graftals' by Smith (1984), who considered them to be a generalization of 'fractals'. When represented as 3D structures they are strikingly 'life-like' (see Fig. 1, Hogeweg and Hesper, 1974; Smith, 1984; Prusinkiewicz and Lindenmayer, 1990). One should, however, keep in mind that such a $3 \mathrm{D}$ representation is entirely a post-
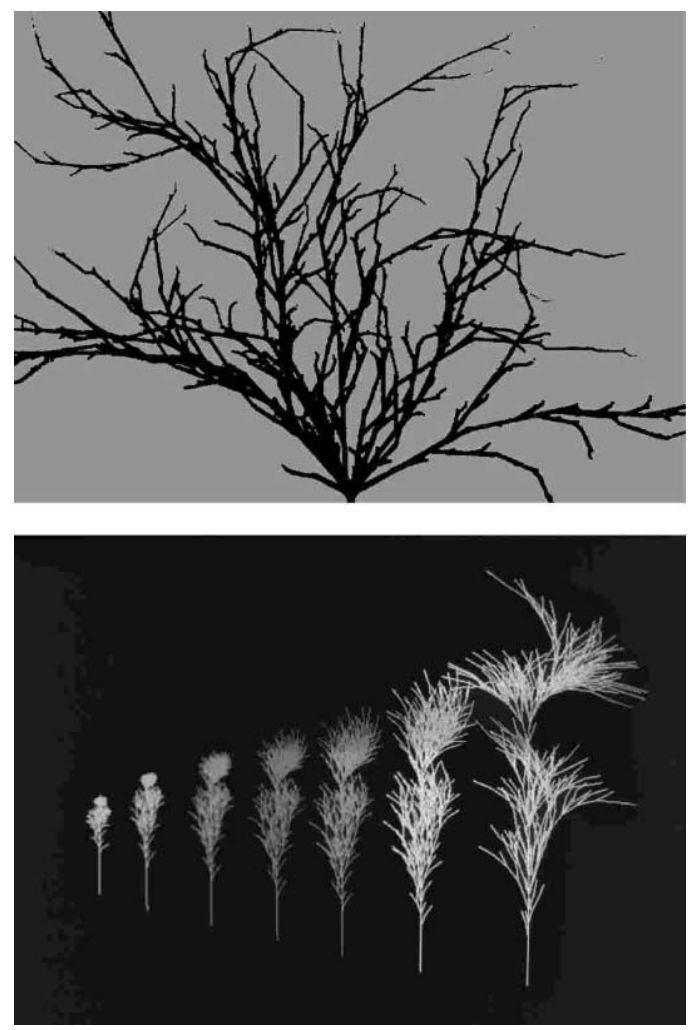

Fig. 1. 3D representation of a 2-state bracketed L-system by post processing. Upper panel: by manipulating e.g. thickness of branches a very life-like pattern is obtained (image courtesy of AR Smith). Lower panel: manipulation of branch length of one stage of the development suggests a developmental sequence. processing step. The impact and flexibility of the post-processing step is illustrated in Fig. 1b: the entire series of pictures does not represent 'development' in the sense of L-systems - they all represent the same stage! The differences are due to the varying length of the branches and the angle of the branch relative to its lower order branch: while these decrease from lower to higher-order branches in the left most patterns, they increase in the right-most patterns.

We conclude that in both these classical approaches to modeling 'development' there is a strong separation between 'development' as modeled, and the actual form that arises. In contrast, in the following sections we will develop a modeling approach that allows us to combine a spatial embedding with a growing cell-based system. Such a system allows us to study gene regulation, cell differentiation, inter-cellular signaling cell growth, cell division and cell death, and the actual morphogenesis that is the result of the interaction between all these processes.

\section{Modeling approach: two scales and beyond}

The core of the models I use in the next two sections is the Glazier and Graner (1993) model for cell sorting. They developed the model as an extension of the Large Q Potts model. In the model, a biological cell (here after called cell) is represented by a number of cellular automaton cells (here called sites) with the same state. Interaction between the cells is based on an 'energy' (H) minimization rule that operates at the scale of the sites but includes variables and parameters that are defined for cells. Most notably, the minimization term includes a cell volume conservation term and the energy bonds $\left(J_{\mathrm{ij}}\right)$ are defined at the level of the cells as well. At each time step, $N$ times a site is selected randomly, and so is one of its neighbors. $\Delta H$ is calculated if the site would copy the state of the neighboring site and the probability of update is determined using the Boltzmann equation. Thus cells expand and retract 'pseudo podia', can be deformed, grow and shrink, and can therefore, squeeze along each other: all these are features that are very hard or 
impossible to represent in models defined on a single scale.

Apart from its intrinsic versatility, the beauty of this model is in the ease with which it can be interfaced with other processes in such a way that the interaction between the processes gives rise to novel phenomena. In the next two sections we present two such extensions. In Section 3 we study the interaction between excitable media and this cell sorting model. We show that large parts of the development of cellular slime molds can be modeled from just these two ingredients, once the model parameters are set. Very little 'interference' by genes is necessary to form a functioning multicellular organism from a random assemblage of single cells. In contrast, in Section 4 we study the interaction of gene regulation and cellular adhesion. We do not do so by trying to mimic an existing organism, but we evolve 'critters' and study the morphogenetic mechanisms that ensue, as well as the evolutionary process itself. We show that it is indeed the interaction of gene regulation and cell adhesion that shape both morphogenesis and evolution.

\section{Computing an organism: 'from single cells to slender stalk'}

Dictyostelium discoideum (Dd) is an organism with a curious life-cycle, which is often, used as paradigm for studying minimal conditions that can generate functional multi-cellularity. They live and feed as unicellular amoeboids; when food becomes scarce they aggregate into a mount, the mount topples over and crawls away as a slug, guided by light and temperature gradients. At a suitable spot it settles once more and develops into a fruiting body that has the form of a slender stalk with a spore mass on top. Cell sorting in the mount and the slug stage results in spore cells in the back/bottom of the cell mass, and prestalk cells in the front/tip. The formation of the fruiting body thus involves complex cell movements that lead to a reversal of this positioning.

Within one-and-the-same modeling framework we have been able to produce (almost) the entire development of Dd (Savill and Hogeweg, 1997;
Marée et al., 1999a,b; Marée and Hogeweg, 2001). An overview of the results is given in Fig. 2. The model incorporates some experimentally well-established properties of Dd., i.e. differential cell adhesion, and long range cell signaling. The latter takes the form of an excitable medium: cAMP release is triggered by cAMP. It is modeled by a generic PDE model for excitable media and we use a discretisation corresponding to the sites of the above described GG model of cell adhesion. Chemotaxis of the amoeboids up the cAMP gradient is modeled on the scale of the sites in the GG model: extension of a phillopodia in the direction of the gradient is (periodically) more likely. The initial condition is a random distribution of single cells of two types (prestalk and prespore cells) which differ in the surface energy parameters only. A subset of the prestalk cells, produces cAMP autonomously-the other cells relay the signal and all cells respond chemotactically to the signal. From these ingredients the aggregation (Fig. 2 upper panel), orientation (Fig. 2 middle panel) and culmination (Fig. 2 lower panel) into a fruiting body unfold. We here comment on the features which emerge due to interaction of the excitable medium and cell adhesion (for more details see the above mentioned publications).

- Aggregating amoeboids form streams (Fig. 2b). Due to the cell adhesion, stream formation increases the speed of the aggregation. Single cells only move up the gradient during a short period. Due to 'pushing' and 'pulling' of other cells (caused by the adhesion and volume conservation) the period of directed motion is extended in the streams.

- In the mount and slug stage the prestalk cells sort out to the top/front and prespore cells at the bottom/back of the cell mass (Fig. 2a-e) The question whether this is caused by chemotaxis or adhesion is hotly discussed in the literature (see e.g. the recent paper of Clow et al. (2000). Our model shows that this 'either-or' question is ill-conceived: the two 'causes' enhance each other. No difference in chemotaxisstrength is needed, but chemotactic motion is needed to accomplish the cell sorting in the correct time frame. 


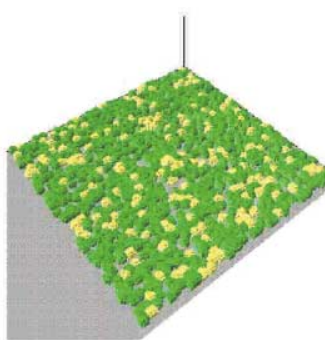

(a)

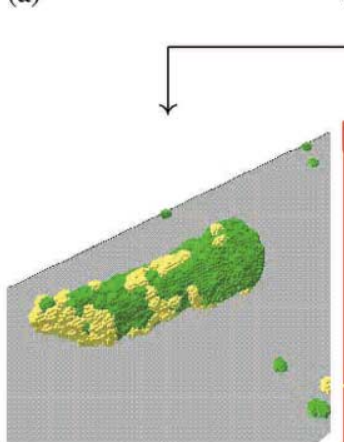

(e)

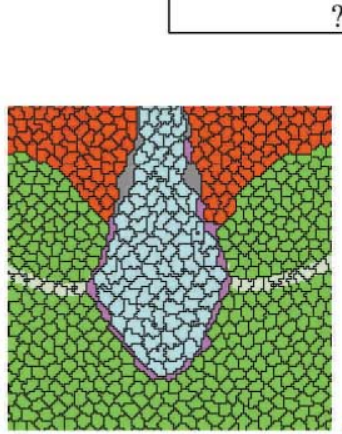

(i)

(j)

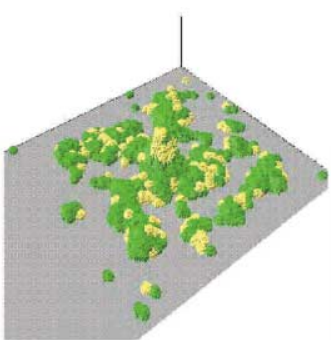

(b)

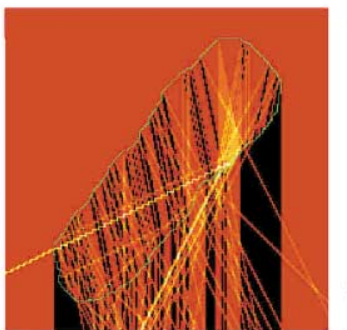

(f)

$?$

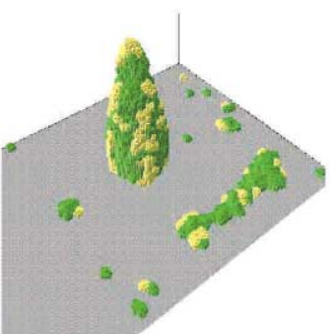

(c)

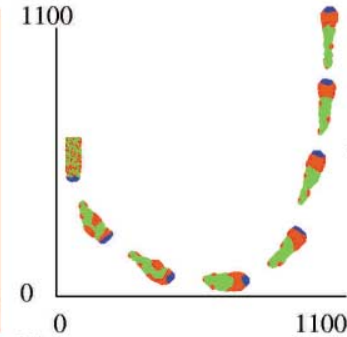

(g)

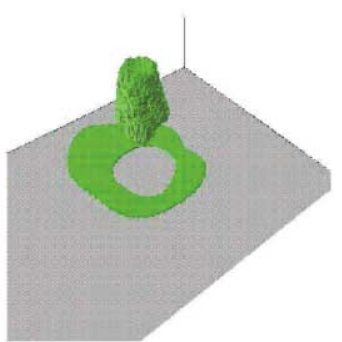

(d)
1100

(h)
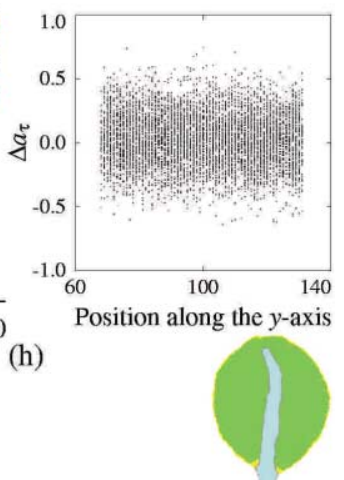

Fig. 2. The development of Dictyostelium in a model combining cell adhesion and cell signalling, (a)-(e) from single cells to crawling slug; (f) the 'lens effect' focusing the light; (g) orientation of the slug towards the light; (h) Dd will show thermo-taxis even in such noisy gradient; (i) the stalk cells are squeezed down by the pressure waves generated by the periodically upward motion of the (pre)spore cells; (j) the formation of a slender stalk with a spore mass on top.

- Orientation of the slug towards light is caused by an intricate interplay between the shape of the excitation waves, the chemotaxis, and momentum caused by cell crowding. Light is focussed at the opposite site of the cell mass, because of the different breaking index of the cells and the 'outside' (Fig. 2f). Increased light causes the production of ammonia (modeled by another PDE); $\mathrm{NH}_{3}$ decreases the sensitivity of the cells toward cAMP, the waves become skewed, and the slug as a whole orients toward the light source, even if it starts out in the opposite direction (Fig. 2g).

- The orientation is very robust. In the case of a temperature gradient (also mediated by ammonia production) we studied the influence of noise on a very shallow gradient. Even in the case shown in Fig. $2 \mathrm{~h}$ almost all slugs do turn into the direction of the (barely visible) gradient. This robustness is caused by the informa- 
tion integration in the waves (which remain straight) and again the crowding of the cells.

- During the culmination phase the prestalk cells differentiate into stalk cells, which move towards the bottom and anchor. The prespore cell move upwards to form the spore mass. The upward movement of the spore cells can easily be understood in terms of, once again, the chemotaxis toward cAMP that is still being signaled by auto-cycling prestalk cells at the top. We show in our model that, indirectly the downward movement is caused by this signal as well! Stalk cells do not relay and do not respond to cAMP, and produce a stiff extra cellular matrix. They are squeezed down due to the periodic pressure waves caused by the upward movement of the prespore cells (see Fig. 2i) provided that a small group of stalk cells at the tip is not surrounded by the stiff slime. The combination of upward and downward movement produce the so called 'reverse fountain' that transforms a heap of cells into a slender stalk with a spore mass on top (Fig. 2j).

- Also the stalk formation is very resistant to noise. The stalk will go straight down, correcting any initial misalignment through different arrival times of the pressure waves (data not shown).

Note the two question marks in Fig. 2. The seemingly 'easy' step of toppling over of the mount is not yet understood in our model, (or in experiments: it is not simply caused by gravity). The transition from slug to the culminant is also not yet modeled. Note also the relative minor role of changes in gene expression in the model: most of the process unfolds without assuming changes is the behavior or parameters of the individual cells. Only during the culmination phase do we model a simple 'maturation' process: auto-cycling prestalk cells become stalk cells, and relaying prestalk cells become auto-cycling prestalk cells; both these maturation steps are triggered by adjacency to these cell types.

We conclude that a functional multicellular critter, and its repertoire of observations and reactions of the environment can be the result of the interaction between two well studied simpler 'modules', i.e. the cell adhesion model and cell signaling as excitable media. In modeling, both these processes we ignored much biological detail, but we nevertheless, uncovered the importance of entanglement of various processes.

In the next section, we will focus on the interaction between gene regulation and cell adhesion, i.e. we will study how genes can assume a more 'active' role than setting of the parameters of an otherwise (almost) autonomously unfolding process as is the case in the model presented in this section.

\section{Evolving morphogenesis: the interplay between cell adhesion and cell differentiation}

Whereas, in the previous section we modeled an existing organism, using processes known from experiments, in this section, we do not try to mimic any particular organism. Instead we try to uncover some generic features of evolving and developing systems, and the interplay between inherited information (genes and gene expression) and the physical dynamics of the system. We do this by studying these issues in critters which we evolve using a very general fitness criterion: the studied features are the side effects of the evolution rather than its 'goal' (see Hogeweg, 1998 for a discussion of this strategy to uncover 'generic nongeneric' features of complex systems).

\subsection{The model}

An overview of the model is shown in Fig. 3. It combines the GG model with a Boolean gene regulation network in each cell, and an evolutionary process to focus on networks which generate morphogenesis. For a detailed description of the model see Hogeweg (2000a). Development starts with one large cell that undergoes a number of prescheduled cleavages; during the first two cleavages 'maternal factors' may cause cell differentiation. The rules for subsequent cell growth and division are based on the experimental observation that stretch can indeed lead to cell growth and division and squeezing of cells leads to apoptosis (Chen et al., 1997; Ruoslahti, 1997). Note that both these processes involve an elaborate 
sequence of changes in gene expression; we here implement only the effect. Cell division occurs in the model when a cell has reached a size that is twice some reference size, and cell division is through the middle and perpendicular to the longest axis. This seems indeed to be the default situation, and modeling of the spindle formation has shown that it will self organize accordingly (Dogterom et al., 1995). However, in vivo many mechanisms are used to alter the division plane. We will examine below their effect on developmental robustness.

In earlier work (Hogeweg, 2000a,b, 2001) we have shown:

- differentiation into cell types varies between (a) stable differentiation, i.e. differentiation is maintained independent of inter-cellular signaling, (b) history dependent differentiation, in which the cell type does dependent on a sequence of neighborhood condition, and (c) differentiation which is fully specified by the current neighborhood only. Each of these types of differentiation is associated with different types of morphogenesis.

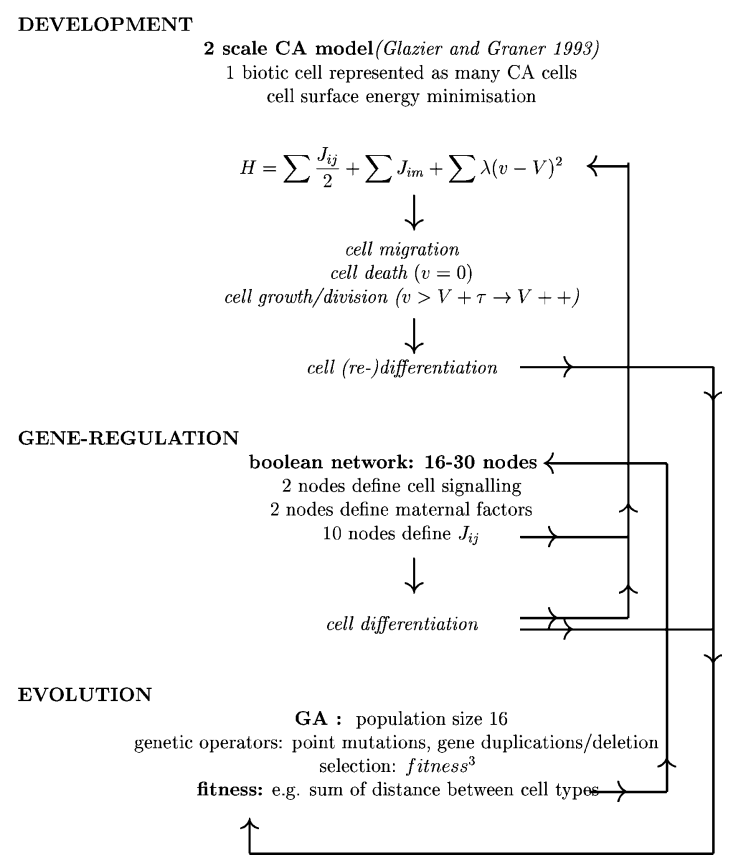

Fig. 3. Overview of the model: entanglement between gene regulation, development and evolution.
- Morphogenesis results as 'sustained transient' from surface energy minimization and 'intrinsic conflict', which is maintained by cell differentiation, cell growth and cell death. Without continued 'interference', the initial, high energy state would change, through shape changes to, at the end a 'blob'-like low-energy shape.

- These intrinsic conflicts lead to automatic orchestration of adhesion, migration, differentiation, cell growth/division and death. It results in 'pseudo-isomorphic outgrowth'. Although, the shapes do change during 'maturation', a 'critter' preserves its general appearance.

- Many different morphemes result from combinations of a few mechanisms.

- An important mechanism is meristematic growth, a layer of dividing cells that differentiate into non-dividing (or rarely dividing) cells of several types. The zone is maintained because, cell redifferentiate if 'out of line': cell differentiation that is fully dependent on neighborhood is 'used'.

- A related mechanism we dubbed elongation by 'budding': a small group of differentiated cells is pushed outwards, because, an other cell type on the one hand tends to engulf them, but on the other sticks together more firmly than to the 'bud'. Again, the situation is maintained, because, cells which do, nevertheless engulf, differentiate into bud-type cells. Like the previous one, this mechanism depends on neighborhood dependent cell differentiation. The elongation shown in Fig. 4 is an example of this.

- Another often occurring mechanism is convergence extension, which occurs due to maximization of the contact line between stably differentiated cell types, and often involves redifferentiation of subtypes of these cell types when the contact zone increases.

- Elongation can also result from intercalation of stably diverged cell types, and their subsequent growth and division.

- Finally engulfing is an intrinsic mechanism of differential cell adhesion. In our model it often induces neighborhood dependent cell differentiation. 

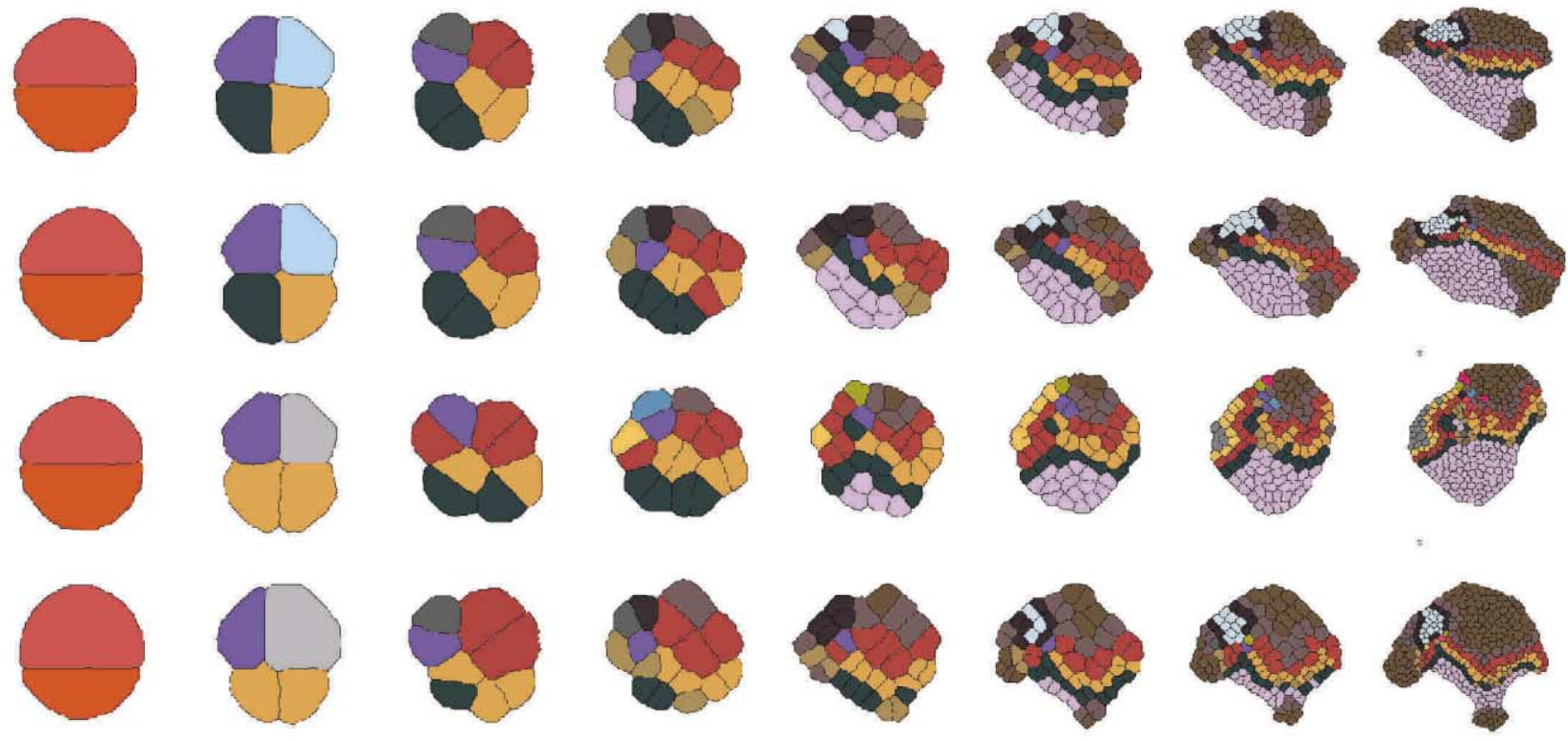

\section{Developmental Variability}

Frequency of occurence of cell types
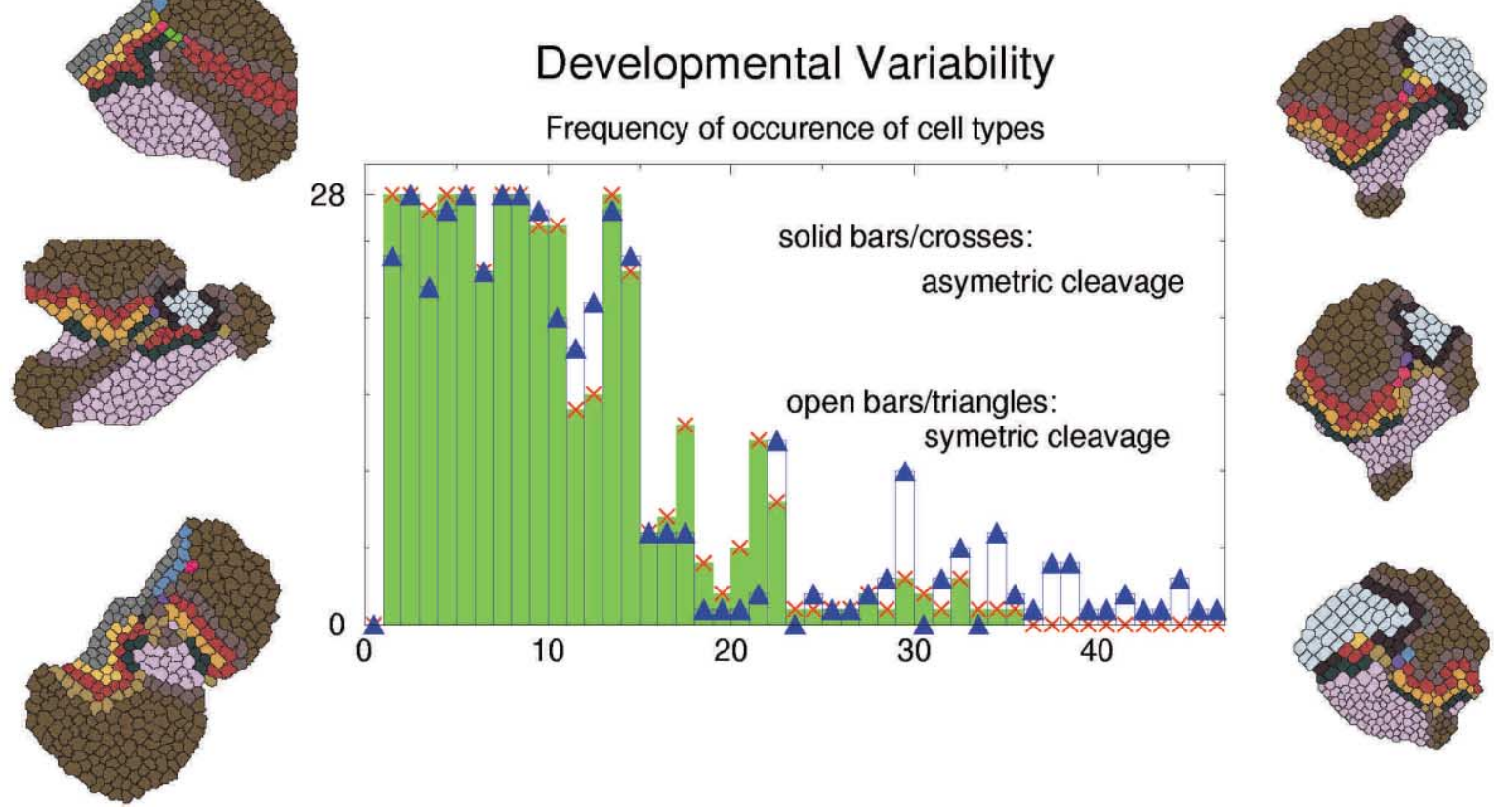

Fig. 4. Individual variation in the phenotypes developed from the same genome and its partial stabilization by asymmetric early cleavage Upper panels: developmental sequence Stages shown just after cleavage and cell redifferentation. Left hand morphemes: variants developed under symmetric cleavage. Right-hand morphemes: variants developed under symmetric cleavage. Histogram shows number of occurrences of cell types as indicated. 
- The evolutionary dynamics shows many of the features known to result from a non-linear genotype-phenotype mapping, i.e. neutral paths and punctuated equilibria at the phenotypic level, although, the shape of the quasispecies distribution differs from the simpler examples studied before (e.g. Huynen et al., 1996; van Nimwegen et al., 1999). We will below examine this in more detail.

- Moreover, the evolutionary dynamics gives rise to interesting mosaic like variation at the phenotypic level, i.e. repeated reinvention of similar morphotypes occurs in one evolutionary history.

All these features involve the interaction of the intra- and inter-cellular levels, which generate long range correlations. In the next two sections we will examine the relationship between the 'informatic' processes, i.e. gene regulation, and the dynamic processes, i.e. cell behavior due to differential adhesion, by focusing on the morphological variation that occurs among critters with identical genomes. In other words we will study how the genetic and inheritable information can on the one hand exploit and on the other hand 'tame' the dynamics of the system.

\subsection{Developmental variability and robustness: the role of asymmetric cleavage}

Developmental robustness seems to be an important feature for organisms, at least for all properties contributing to its fitness. In our model, gene expression is deterministic but dependent on signals of neighboring cells. In contrast, cell adhesion, and the resulting changes in shape of the cells and cell movements, growth and cell death are stochastic. Through cell neighborhood they can change gene expression, which in its turn may affect cell adhesion parameters. In this way, small stochastic differences can be blown up and quite different critters may develop from identical genomes. The positive feedback is in fact required to obtain and maintain interesting shapes despite the circular initial conditions and the tendency of the minimization rule to produce 'blobs'. The mechanisms of morphogenesis described above all depend on it. Here we ask the question: In what way can and/or does the inheritable information harness the physical dynamics in such a way that the positive feedback leads to similar critters, which, nevertheless do not simply correspond to the attractor of the 'physical' dynamics?

Fig. 4 shows an example of a genome that can lead to critters with quite different shapes, and also with different cell types. The upper three rows show the development of three of these, and other examples of 'mature' critters are shown in the lower part of the figure. From the developmental sequences we see that a source of variation in the final critter is slight variation in the division planes in early (or somewhat later) cleavage division. This leads to variation in cell contacts, and therewith in gene expression and therewith in the adhesion forces and resulting cell shape, which will lead to further differentiation in cell contacts. Note, however, that in many respects convergence of the critters and cell types also occur. Note for example the occurrence of green and grey cell types at different stages of the development.

It is well known that the location and direction of the division plane is precisely regulated in early cleavages (for a recent review see Nishida et al., 1999). Successive cleavages are usually perpendicular and asymmetric division leading to macromeres and mircromeres is seen in many species. The tight regulation supposedly serves to capture cytoplasmatic localized factors in one daughter cell only. Indeed, regulation of localization of maternal factors and of the division plane seem to be coupled directly. A second 'purpose' seems to be the determination of cell contacts. Interestingly, several types of regulation of the division plane operate in different organisms and even in different stages of the same organism. This suggests that it evolved repeatedly and suggests import selection pressures to do so.

Here we study how asymmetric division alters the variability observed in the critter displayed in Fig. 4. In our model maternal factors are assumed to be captured perfectly in any case. We limit asymmetry however, to the first two cleavages, i.e. the ones in which cell differentiation due to maternal factors occurs in our model. Thus we investigate the role of determining cell contacts in the first divisions on the long term development. 
The fourth row of Fig. 4, displays the development of the critter with asymmetric division. The asymmetric division limits cell contacts after the second division to correspond to those in the third row which occurs in $50 \%$ of the cases in the symmetric case. However, influence of asymmetric division appears to extend beyond this stage. It is interesting to see the similarity of the cell types in the third stage: the same cell types occur in row 1 , 2,4 , while row 3 differs. In later stages, due to the size differences of the cells, the cell deformation and movement is more constrained than it is in the default, symmetric case.

In the histogram we show that not only shape differences are reduced due to the asymmetric division, but also variation in cell types is reduced. There are more cell types that occur always for development with asymmetric first and second cleavage than for symmetric first cleavages. Moreover, many more cell types occur one or a few times in the set generated by symmetric cleavage than that generated by asymmetric cleavage. We conclude that asymmetric cell division in early stages indeed seems to be an efficient strategy to exploit the potential of cell interactions for early differentiation, while limiting the amount of developmental variation.

In the next section, we demonstrate the tendency of evolutionary dynamics to minimize developmental variation and mutational sensitivity. We do this in the standard model with symmetric cell division.

\subsection{Evolution of developmental and mutational robustness}

In Fig. 5, upper panel, the fitness of an evolving population is plotted. The dots represent the fitness of all members of the population, the filled circles are the common ancestors of the final population. In this case, we use a pseudo-morphological criterion for fitness, instead of our usual fitness criterion on number and differentiation of cell types. The criterion is 'total path length' of identical cell types, i.e. selection favors long layers of cells of the same type (maximum of two layers per cell type are counted), as well as many cell types. In fact during the entire period shown in the figure the 'strategy' for maximizing this criterion remains similar: concentric cell layers develop using engulfing, cell type induction, and maximization of the interface between layers.

The evolutionary dynamics shows the following interesting features:

- Two types of stepwise improvement occur:

$\circ$ at time $=479$, a new phenotype is discovered that has much higher fitness than the previous ones. The phenotypic transition is shown in the two pictures on the left. The new genotype takes over the population very rapidly: the two vertical lines show that the entire population on the right is ascended from one-and-the-same individual on the left line.

- Despite the rapid take-over, there is only a very temporary increase in the median fitness of the population (red line) and a slight increase in the average fitness. At around time $=900$, however, a stepwise increase in median (but not average) fitness occurs. The previous discovered phenotype is stabilized both developmentally and mutationally. Nevertheless, aberrant development still does occur. The common ancestor at time $=1308$ is an example: it has a fitness of 46 and only three cell types. Nevertheless, its genotype is identical to its ancestor, which has six cell types and fitness 83 . The corresponding phenotypes are displayed on the right.

- In the period that neither the maximum fitness, the fitness of the common ancestors, the median or the average fitness changes, the shape of the quasi-species still does change. In the later period, there are more individuals with very high fitness, and very low fitness, as shown in the histogram of Fig. 5. Still aberrant development does occur, however, their fitness remains fairly high.

- The shape of the gene regulation network changes drastically. The left network is the one at time $=479$, the right one is time $=2145$; the corresponding critters are displayed just above the networks. The expression of more genes is unregulated in later stages of the evolution and the network has become much shorter. Thus mutational changes have less effect. 


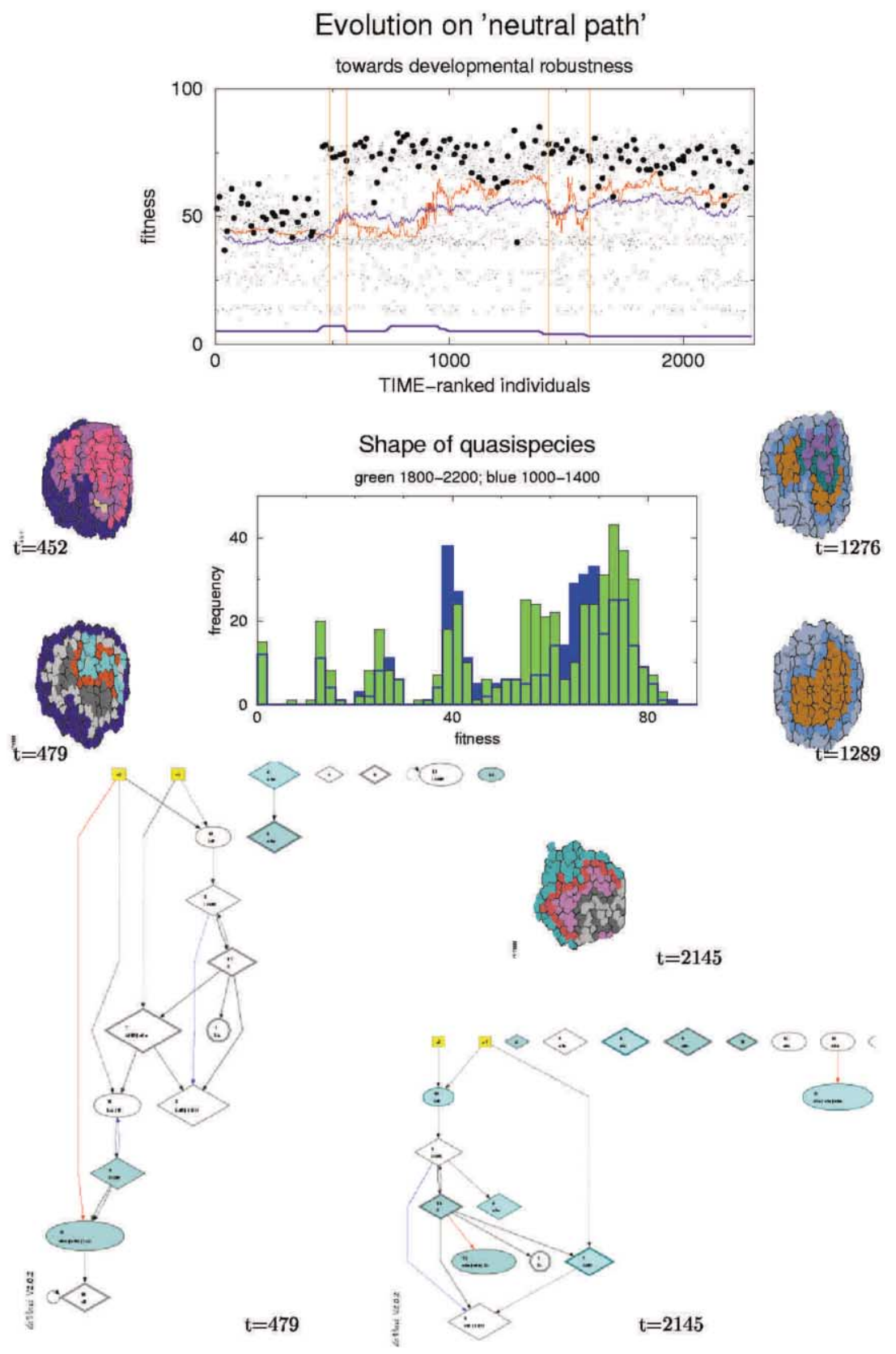

Fig. 5. Evolution of developmental and mutational robustness. Upper panel: distribution of population fitness over time. Black circles represent the common ancestors of the last population; vertical lines: duration of fixation: Red line: medium fitness; Purple line: average fitness. Lower grey line maximum path length in gene regulation network. Middle panel; Histograms of quasi-species distribution in two periods of neutral evolution: distribution change towards higher developmental and mutational robustness. Phenotypes Left transition to high fitness; right developmental variation of identical genotypes. Lower panel change in regulatory network during 'neutral evolution'. The shortening increases the mutational robustness. 
We conclude that the simple evolutionary dynamic implemented does more than simply select fit individuals. Developmental and mutational stability are strongly selected as well. Selection to robustness biases the networks to short ones. It is interesting to investigate this feature in 'real' gene regulation networks when data become available.

The developmental instability of the critters of this evolutionary run is again through differences in the first cell division. Unequal early cleavage should, therefore be strongly selected (were it made possible in our experiments).

Selection for mutational robustness has been shown to be a hallmark of evolution dominated by neutral paths (van Nimwegen et al., 1999) The two types of stepwise evolution, i.e. in maximum and in medium fitness is not yet reported (as far as I know). It is an evolutionary signature of developmental instability.

\section{Discussion and conclusions}

In biotic systems, processes do not operate in isolation. The existence of entanglement of processes at different time and space scales does not need an explanation: it is there by default; ignoring the entanglement, by artificially separating space and time scales is only (a frequently used) modeling artifact.

In this paper, we have examined modeling approaches that do not incorporate this artifact. The aim is to uncover the consequences of the entanglement. Our approach is to study the features that emerge by combining two or more simple descriptions of fairly well known processes, rather than to elaborate the description of these processes by including details of their implementation in biotic systems. By using the relatively simple descriptions and therewith limited number of parameters, we can focus on and study the features that arise through the interplay between these processes. We have shown that through the interplay of the processes we indeed can model not only 'generic' features, which biological systems share with many other systems, but can explain specific, experimentally observed, but previously not yet understood behavior of particular organ- isms (e.g. orientation and culmination in Dictyostelium).

A hallmark of biological systems is that, they are physical dynamical systems, which have evolved. Nevertheless, evolution is seldom studied in interaction with 'physical' systems' with a dynamics of their own'. In most models, genes are supposed to 'do what they do', or the physical systems are supposed to 'do what they do'. The differential adhesion based model of cell sorting with a genetic network governing adhesion parameters proved to be an interesting test-bed for studying the relation between the physical dynamics of the system and the inherited information.

We have seen that, although, the development of the critters is 'governed' (through the cell adhesion parameters) by the gene regulation network, inheritance of phenotype does not follow automatically. The positive feedback between gene expression and adhesion is crucial for the genes to do anything at all, but also causes sensitivity to noise. We have seen, however, that the evolutionary process will reduce this sensitivity, while exploiting the self-correcting features to which the interaction between gene expression and differential adhesion can also give rise. An efficient general way to do so seems to be the unequal cell division seen in the early cleavages of most organisms. We should note, however, that further studies are necessary to generalize the results obtained so far.

In conclusion: entanglement of processes at different time and space scales is a source of interesting, complex and life-like behavior, and should not be ignored in our attempts to model organisms. Evolution under an invarying selection regime automatically leads to increase of robustness against mutations (Huynen and Hogeweg, 1994; van Nimwegen et al., 1999) and against developmental variation (this paper). We have shown that it is feasible to formulate models that incorporate and exploit these two facts. This is done by allowing (but not rigorously predefining) entanglement of processes, and by using an evolutionary processes to automatically tune positive and negative feedbacks between levels, as a side effect of some general fitness criterion. Thus, we come one step closer to, in some sense, 'computing an organism' although, some of the desiderata 
of computing systems (e.g. full modularity and environmental independence) are certainly not met (or desired) in these models.

\section{Acknowledgements}

I thank Nick Savill and Stan Marée for their research on Dictyostelium development, and the latter also for composing fig. 2. I am much indebted to Roeland Merks for developing the computer program for the evolution for morphemes. I thank Ben Hesper for long-term discussions and support.

\section{References}

Chen, C.S., Mrksich, M., Huang, S., Whitesides, G.M., Ingber, D.E., 1997. Geometric control of cell life and death. Science 276, 1425-1428.

Clow, P.A., Chen, T., Chisholm, R.L., McNally, J.G., 2000. Three-dimensional in vivo analysis of Dictyostelium mounds reveals directional sorting of prestalk cells and defines a role for the myosin II regulatory light chain in prestalk cell sorting and tip protrusion. Development 127 (12), 2715-2728.

Conrad, M., 1995. Cross-scale interactions in biomolecular information processing. Biosystems 35 (2-3), 157-160.

Conrad, M., 1996. Cross-scale information processing in evolution, development and intelligence. Biosystems 38 (2-3), 97-109.

Dogterom, M., Maggs, A.C., Leibler, S., 1995. Diffusion and formation of microtubule asters: physical processes versus biochemical regulation. Proc. Natl. Acad. Sci. USA 92 (15), 6683-6688.

Glazier, J.A., Graner, F., 1993. Simulation of the differential driven rearrangement of biological cells. Phys. Rev. E 47, 2128-21254.

Hogeweg, P., 1998. On searching generic properties in nongeneric phenomena: an approach to bioinformatic theory formation. Artificial Life VI (e.s C. Adami, R.K Belew, H. Kitano and C.E. Taylor MIT press pp. 285-294.

Hogeweg, P., 2000a. Evolving mechanisms of morphogenesis: on the interplay between differential adhesion and cell differentiation. J. Theor. Biol. 203 (4), 317-333.

Hogeweg, P., 2000b. Shapes in the shadow: evolutionary dynamics of morphogenesis. Artif. Life 6 (1), 85-101.

Hogeweg, P., 2001 Multilevel processes in evolution and development: computational models and biological insights In: Michael Laessig and Angelo Valleriani (Eds.) Biological Evolution and Statistical Physics, Springer, in press.
Hogeweg, P., Hesper, B., 1974. A model study on morphological description. Pattern Recogn. 6, 165-179.

Huynen, M.A., Hogeweg, P., 1994. Pattern generation in molecular evolution: exploitation of the variation in RNA landscapes. J. Mol. Evol. 39, 71-79.

Huynen, M.A., Stadler, P.F., Fontana, W., 1996. Smoothness within ruggedness: the role of neutrality in adaptation. Proc. Natl. Acad. Sci. USA 93, 397-401.

Kerszberg, M., Wolpert, L., 1998. Mechanisms for positional signalling by morphogen transport: a theoretical study. J. Theor. Biol. 191 (1), 103-114.

Lindenmayer, A., 1968a. Mathematical models for cellular interactions in development. I. Filaments with one-sided inputs. J. Theor. Biol. 18 (3), 280-299.

Lindenmayer, A., 1968b. Mathematical models for cellular interactions in development. II. Simple and branching filaments with two-sided inputs. J. Theor. Biol. 18 (3), $300-315$.

Lindenmayer, A., 1975. Developmental algorithms for multicellular organisms: a survey of L-systems. J. Theor. Biol. 54 (1), 3-22.

Marée, A.F., Hogeweg, P., 2001. How amoeboids self-organize into a fruiting body: multicellular coordination in Dictyostelium discoideum. Proc. Natl. Acad. Sci. USA 98 (7), 3879-3883.

Marée, A.F., Panfilov, A.V., Hogeweg, P., 1999a. Migration and thermotaxis of dictyostelium discoideum slugs, a model study. J. Theor. Biol. 199 (3), 297-309.

Marée, A.F.M., Panfilov, A.V., Hogeweg, P., 1999b. Phototaxis during the slug stage of Dictyostelium discoideum: a model study'. Proc. R. Soc. London B 266, 1351-1360.

Meinhardt, H., Gierer, A., 2000. Pattern formation by local self-activation and lateral inhibition. Bioessays 22 (8), $753-$ 760 .

van Nimwegen, E., Crutchfield, J.P., Huynen, M.A., 1999. Neutral evolution of mutational robustness. Proc. Natl. Acad. Sci. USA 96, 9716-9720.

Nishida, H., Morokuma, J., Nishikata, T., 1999. Maternal cytoplasmic factors for generation of unique cleavage patterns in animal embryos. Curr. Top. Dev. Biol. 46, 1-37.

Prusinkiewicz, P., Lindenmayer, A., 1990. The Algorithmic Beauty of Plants. Springer, New York.

Ruoslahti, E., 1997. Stretching is good for a cell. Science 276 (5317), 1345-1346.

Salazar-Ciudad, I., Sole, R.V., Newman, S.A., 2001. Phenotypic and dynamical transitions in model genetic networks. II. Application to the evolution of segmentation mechanisms. Evol. Dev. 3 (2), 95-103.

Savill, N.J., Hogeweg, P., 1997. Modelling morphogenesis: from single cells to crawling slugs. J. Theor. Biol. 184, 229-235

Segel, L.A., 2001. Computing an organism. Proc. Natl. Acad. Sci. USA 98 (7), 3639-3640.

Smith, A.R., 1984. Plants, Fractals and Formal Languages. Computer Graphics 18, 1-10. 